\title{
Joint Voronoi diagram and game theory-based power control scheme for the HetNet small cell networks
}

\author{
Xiaodong $\mathrm{Xu}^{1 *}, \mathrm{Yi} \mathrm{Li}^{2}$, Rui Gao ${ }^{1}$ and Xiaofeng Tao ${ }^{1}$
}

\begin{abstract}
With the introduction of small cell into current macro cell structure, the ever-growing demand for mobile data service has the opportunity to be fulfilled. But the correspondingly overlaid dense deployment situation caused by the Heterogeneous Network (HetNet) also arouses the interference and mobility management problems. In order to solve the above problems, we propose the Joint Voronoi diagram and game theory-based power control scheme for the HetNet small cell networks with two-step approach. The first step focuses on the optimization of the small cell cluster deployment planning within the coverage of the macro cell. The intra-tier interferences are mitigated by the min-max power allocation algorithm and the mobility management performance can also be improved by the Voronoi diagram-based scheme. Then, the second step addresses on the mitigation of cross-tier interferences while protecting the guaranteed users and high-mobility users usually served by the macro cells. The game theory-based dynamic power control scheme is proposed via non-cooperative game model with the convex pricing function. The existence and uniqueness of the Nash equilibrium for the proposed game model are verified, which provide the feasible solution for the cross-tier power control in the heterogeneous network. From the observation of the system-level simulation results, the proposed scheme can bring a significant increase in the system throughput, reduce the outage probability, as well as enhance the energy efficiency over current works.
\end{abstract}

Keywords: HetNet; Small cell networks; Power control; Voronoi diagram; Game theory

\section{Introduction}

Faced with the exponentially increasing demand for mobile data services, the cellular network operators are confronted with a precarious situation: accommodate the explosive traffic growth yet reduce the costs and consequently increase average revenue per user (ARPU) [1,2]. While the traditional cellular structure mainly including the macro cell has been effective in providing the required capacity for voice and low data rate services, with their large coverage, they are not potent in the provision of higher data rates in the hotspots. Besides, due to the high penetration losses, the macro cells are not suitable for further providing adequate indoor coverage where it emerges more than $70 \%$ future mobile data services [3].

\footnotetext{
* Correspondence: xuxiaodong@bupt.edu.cn

${ }^{1}$ National Engineering Laboratory for Mobile Network Security, Beijing University of Posts and Telecommunications, Beijing 100872, China Full list of author information is available at the end of the article
}

Fueled by the needs of high data rate traffic and improved coverage for home and office use, the Heterogeneous Network (HetNet) with small cell network (SCN) deployment have attracted significant interests in the wireless industry and standard organizations [4]. The HetNet with SCN will be a radically different network design that could provide a cost- and energy-efficient solution to cope with the forecast traffic growth. SCN is founded by the idea of a very dense deployment of self-organizing, lowcost, low-power base stations that are substantially smaller than the traditional macro cell equipment [5]. The small cells could be installed on the building walls or urban furniture, in the streets, or inside the hotspot buildings. They are expected to offload part of capacity from the macro cell, thereby reducing the capital expenses (CAPEXs) and operational expenses (OPEXs) for the network operators. In addition, the transmission range of small cells can offer better signal reception at indoor terminals. Although 
SCNs can provide significant benefits, they may suffer severe interference with the high-density heterogeneous deployments, emanating from other nearby small cells and the macro cells with the shared spectrum manner. Moreover, with the optimized cell radius of the small cell cluster deployment planning in the HetNet, user mobility also becomes difficult to handle in the HetNet SCNs and thus raise outage challenges [6].

In order to solve the interference and mobility problems, which are the key challenges for the HetNet small cell network deployment, there have been several researches focused on these areas so far. A class of energy-efficient sleep mode algorithms for small cells is introduced in [2] and [7]. However, there is no priority in interference handling problems. Ericsson and ST-Ericsson [8] proposes a utility-based distributed algorithm to allocate resources yet with no mobility management considerations taken into consideration. In [9], the interferences are managed by a decentralized approach based on multi-armed bandit solutions and the mobility performances are evaluated relying only on local user measurement reports. Game theory has also been used for the power control-based interference mitigation in the heterogeneous networks in [10-14]. In [10], a power control scheme based on game theory is studied for the HetNet. In [11] and [12], the Stackelberg game model and repeated game model are studied respectively for two-tier femtocell networks, which is a typical example for the scenario of macro cells with shared spectrum small cell networks. In [13] and [14], linear convex pricing-based non-cooperative game was applied for the power control schemes, but the deployment optimization of the small cell cluster and user mobility are not considered.

Motivated by the limitations of the current literature, in this article, we researched the power control for the interference and mobility management of the macro cell and small cell heterogeneous deployment scenario. The proposed power control scheme includes two steps:

a) At first, we mainly consider the small cell cluster deployment within the coverage of macro cells. The interferences between the macro cell tier and the co-located small cell tier are ignored temporally. In order to have the optimal deployment of small cell base stations (BSs) with lower energy cost and outage probability while guaranteeing the coverage of the small cell clusters, we propose the Voronoi diagram-based power allocation scheme to obtain the minimizing transmission power limitations, $p_{\max }\left(B S_{i}\right)$, for each small cell, which is named min-max power allocation algorithm.

b) After getting the deployment of small cell cluster via the min-max power allocation algorithm, we propose the game theory-based power control scheme for further maximizing the throughput and mitigating the cross-tier interference with taking into consideration of co-channel interferences within the macro cell and small cell heterogeneous deployment. The transmission power of each small cell BS will be deduced by the actual distribution of users and interferences via non-cooperative game model, which varies from 0 to the $p_{\max }\left(\mathrm{BS}_{i}\right)$ obtained by the min-max power allocation algorithm.

The contributions of this article include several aspects. Firstly, the Voronoi diagram-based deployment planning optimization approach for the small cell clusters within the HetNet could solve the intra-tier interference problem by minimizing the maximum transmission power of densely deployed small cells. The coverage hole and cross-coverage area are optimized based on the Voronoi diagram. Therefore, the cell edge user throughput and system outage probability are reduced, which could improve the user mobility performance. Furthermore, considering the cross-tier interferences, the game theory-based power control scheme with convex pricing functions could mitigate the cross-tier interference while protecting the guaranteed users and high-mobility users usually served by the macro cells. The existence and uniqueness of the Nash equilibrium for the proposed game model are verified, which provides the feasible solution for the cross-tier power control in the HetNet deployment scenario. The system throughput, outage performances, and energy efficiency are observed to be enhanced over current schemes.

The rest of the article is organized as follows. Section 2 describes the system model and illustrated the problems to be solved. The Voronoi diagram-based min-max power allocation algorithm is proposed in Section 3 and based on the results of that, the game theory-based power control scheme is described in Section 4 with the analyses of the game model and Nash equilibrium. In Section 5, the simulation results for evaluating the heterogeneous network performances are provided. The observations and analyses for the results are also presented. Finally, there comes the conclusion.

\section{System model}

According to the 3rd Generation Partnership Project (3GPP) Long Term Evolution-Advanced (LTE-A) standardization, due to the scarcity of available spectrum, the HetNet with small cells will inevitably be deployed in shared spectrum manner. In this article, we will focus on the shared spectrum scenario with consideration of cross-tier interferences. An illustration of the deployment scenario is shown in Figure 1.

We can see that to address the dense deployment scenario for higher traffic, each macro cell area is covered by several small cell clusters, the number of which can 


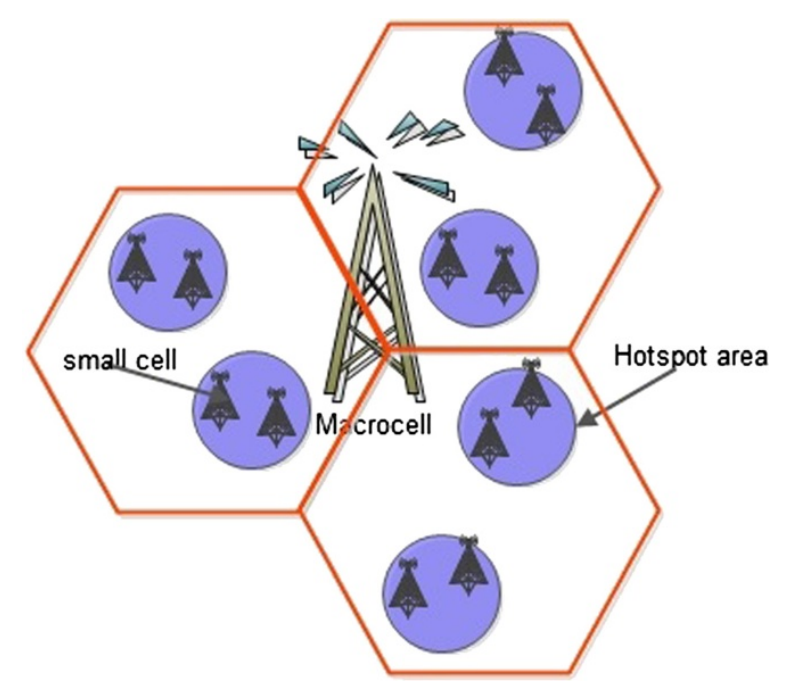

Figure 1 Deployment scenario for the HetNet SCNs.

be 1,2 , or 4 [15]. And each cluster is covered by several small cell BSs, the number of which can be 2,4 , or 10 [15]. Both small cell clusters and small cell BSs are considerably less planed as opposed to the typical macro cell deployments.

We take the downlink scenario of the LTE-A HetNet small cell networks as the research focus. The main responsibility of small cell tier is to offload the high data rate service from the macro cell while the macro cell tier handles the low data rate services and high-mobility users, which is also the actual deployment demand for the HetNet. The service type of the users is full buffer and the throughput performance, outage probability, and system energy efficiency will be the evaluation matrices.

The cross-tier and intra-tier interferences are all considered. But as the first step, we will focus the intra-tier interferences only because we need to get the optimized deployments of small cells by adjusting the maximum transmission power limitation for each small cell BS. Then, based on the obtained power limitation, the crosstier interferences will be mitigated further.

\section{Voronoi diagram-based power control scheme}

As the first step of the proposed joint power control scheme, in this section, our objective is to determine the maximum transmission power limitation for the small cells almost tightly to serve their users. If more power is allocated than demanded, redundant power settings will not only bring more interference to other small cells but also cause immoderate energy consuming. Moreover, users will not be satisfied with their service quality. The improper transmission power limitation setting will also impact the coverage and cross area of each small cell, which will result to worse user mobility management performances. Herein, we propose a Voronoi diagram-based power control scheme.

For the LTE-A downlink, it is an orthogonal frequency division multiple access (OFDMA) system. According to Figure 1, the achievable signal-to-interference-plus-noise ratio (SINR) value on sub-carrier $k$ of a small cell user $j$ served by small cell $i$ can be shown as follows:

$$
\gamma_{i, j, k}=\frac{P_{i, k}^{S-S} H_{i, j, k}^{S-S}}{\sum_{S^{\prime}-S} P_{k}^{S^{\prime}-S} H_{j, k}^{S^{\prime}-S}+N_{0} \Delta f}
$$

where $j=1, \ldots, L, P_{i, k}^{S-S}$, and $P_{k}^{S^{\prime}-S}$ are the transmission powers of the serving small cell $i$ and neighbor small cells on sub-carrier $k . H_{i, j, k}^{S-S}$ is the channel gain between small cell user $j$ and serving small cell $i$ on sub-carrier $k$, while channel gain from neighbor small cell is denoted by $H_{j, k}^{S^{\prime}-S} . N_{0}$ is the white noise power spectral density and $\Delta f$ is sub-carrier spacing.

After calculating $\gamma_{i, j, k}$, a corresponding modulation and coding scheme (MCS) can be assured. When using MSCr, the bit rate $\mathrm{BR}_{r}$ and the throughput $\mathrm{TP}_{i}$ of the small cell $i(i=1, \ldots, N)$ can be modeled respectively as

$$
\begin{aligned}
\mathrm{BR}_{r} & =\Omega \eta_{r}=\frac{C_{\mathrm{OFDMA}} S_{\text {OFDMA }}}{T_{\text {subframe }}} \eta_{r} \\
\mathrm{TP}_{i} & =\sum_{k=1}^{N_{\mathrm{RB}}} \mathrm{BR}_{r}\left(1-\mathrm{BLER}\left(\mathrm{MSC} r, \gamma_{i, j, k}\right)\right)
\end{aligned}
$$

where $\Omega$ is a fixed parameter that depends on the network configuration, and $C_{\text {OFDMA }}$ and $S_{\text {OFDMA }}$ are the numbers of data sub-carriers and symbols per resource block (RB), respectively. $T_{\text {subframe }}$ is the RB duration in time units. $\eta_{r}$ is the efficiency in bits/symbol of $\mathrm{MSC} r$, and $B L E R\left(\mathrm{MSC} r, \gamma_{i, j, k}\right)$ is the block error rate (BLER) of sub-carrier $k$, which is a function of both MSCr and $\gamma_{i, j, k}$.

Assuming $U=\left\{j=1, \ldots, M \| \gamma_{j}<\gamma_{t}\right.$ arg et $\}$ is the set of users that suffer from network outage due to their SINR $\gamma_{j}$ below the target SINR $\gamma_{t}$ arg et. The $\|U\|$ denotes the total number of outage users and the system outage probability can be obtained as follows:

$$
\mathrm{OP}=\frac{\|U\|}{M}
$$

And the system energy efficiency is defined as:

$$
\mathrm{EE}=\frac{\sum_{i=1}^{N} \mathrm{TP}_{i}}{\sum_{i=1}^{N} \sum_{k=1}^{N_{\mathrm{RB}}} P_{i, k}}
$$




\section{Min-max transmission power allocation algorithm}

We first consider an initial power assignment of small cells, the purpose of which is to achieve maximal coverage with minimized maximum required transmission power. As shown in Figure 2a, there may be coverage holes (such as Area 1 in Figure 2a) or large cross-coverage areas (Area 2) if the same fixed transmission power level is assigned to all small cells. Therefore, we propose an adaptive power assignment algorithm to provide a minimum possible transmission power level of each small cell BS. The proposed algorithm can cover a target area and optimize the cross-coverage areas.

Implementing the Voronoi diagram, one well-known geometric structure in computational geometry, we present an algorithm in a two-dimensional (2D) domain with a polygonal boundary [16]. The Voronoi diagram is applied to solve the problem of dividing space into a number of regions. A set of sites is specified for that space beforehand. For each site, there will be a corresponding region consisting of all positions closer to that site than to any other. The regions are defined as the Voronoi cells. For the deployment optimization problem within small cell clusters in the HetNet environment, the Voronoi diagram is quite suitable for planning each small cell BS's coverage via adjusting its maximum transmission power, as long as the positions of each small cell BSs are predetermined as the 'sites' by Voronoi diagram. Moreover, the optimized Voronoi cell will be the corresponding small cell BS's coverage area after the implementation of Voronoi diagram.

In 2D Euclidean space, the Voronoi diagram $V(\mathrm{BS})$ of $N$ small cells is the subdivision of the ichnography into $N$ Voronoi cells. Let $d(a, b)$ be the Euclidean distance between two positions $a$ and $b$. The Voronoi cell $V\left(B S_{i}\right)$ of the small cell $B S_{i}$ is defined as follows:

$$
V\left(\mathrm{BS}_{i}\right)=\left\{x \in \mathbb{R} \| \forall j \neq i, V\left(\mathrm{BS}_{i}, x\right)<V\left(\mathrm{BS}_{j}, x\right)\right\}
$$

where $x$ denotes the positions inside of the Voronoi cell $V\left(B S_{i}\right)$, which means all positions in the $V\left(B S_{i}\right)$ are closer to the small cell $B S_{i}$ than to any other small cell BSs.

Then, the whole Voronoi Diagram for all the sites in the coverage area $V(B S)=\cup_{1 \leq i \leq N} V\left(B S_{i}\right)$.

From the formulae above, we can see that any positions in the Voronoi cell are closer to its Voronoi sites. To minimize the maximum required power of $B S_{i}, V\left(B S_{i}\right)$ should be covered by a small cell located at the site of the Vorono $i$ cell unless the area is covered by other small cells. The first step of our Voronoi diagram based min-max transmission power allocation algorithm is to find the small cell as the Voronoi site whose distance to the farthest position is longest and allocates sufficient transmit power to cover the farthest position as described in Algorithm 1. The farthest position will be one of the vertices of the cell boundary. Assume the communication range is a circle $C(B S, R)$, where $B S$ is the center of the circle and $R$ is its radius. It can be seen from Figure $2 \mathrm{~b}$ that once the maximum transmission power of the current focused small cell $B S_{i}$ is assigned, the Voronoi boundary of its neighbor cells could be adjusted in the following steps according to the already determined coverage area of the small cell $B S_{i}$. As shown in the Figure $2 \mathrm{~b}$, once the maximum transmission power of $B S_{1}$ is determined with the radius $R \_\max \left(B S_{1}\right)$, the $B S_{2}$ could adjust its maximum transmission power for the updated Voronoi boundary from radius $R_{-} \max \left(B S_{2}\right)$ to the Modified $R_{-} \max \left(B S_{1}\right)$. Therefore, we can recalculate the maximum small cell radius of those neighbor sites to minimize the cross-coverage areas. Once the distance $d_{\max }$ $\left(B S_{i}\right)$ is obtained, we can assign the maximum transmission power to the small cell $B S_{i}$ as follows:

$$
P_{\max }\left(\mathrm{BS}_{i}\right)=\min \left\{\mathrm{PL}\left(d_{\max }\left(\mathrm{BS}_{i}\right)\right)+P_{S}\left(\mathrm{BS}_{i}\right), P^{\max }\right\}
$$

where $P L$ represents the path loss. $P_{S}\left(B S_{i}\right)$ is the reference signal received power (RSRP) from the nearest small cell

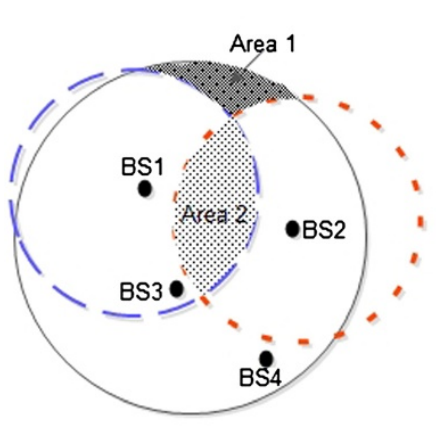

(a) Conventional network deployment

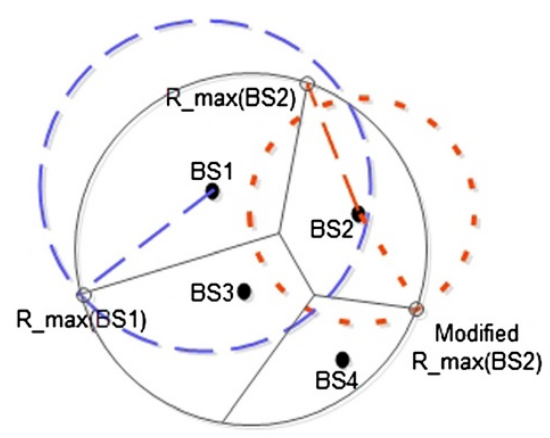

(b) The Voronoi diagram

Figure 2 Min-max power allocation algorithm. 
measured by the current focused small cell and $P^{\max }$ is the radio frequency $(\mathrm{RF})$ transmission power limitation of the small cell BSs. Since the algorithm operates with the site whose distance is currently the maximum at each iteration, it guarantees that the maximum transmission power assigned is minimized. The Voronoi diagram-based minmax transmission power allocation algorithm is introduced as follows.

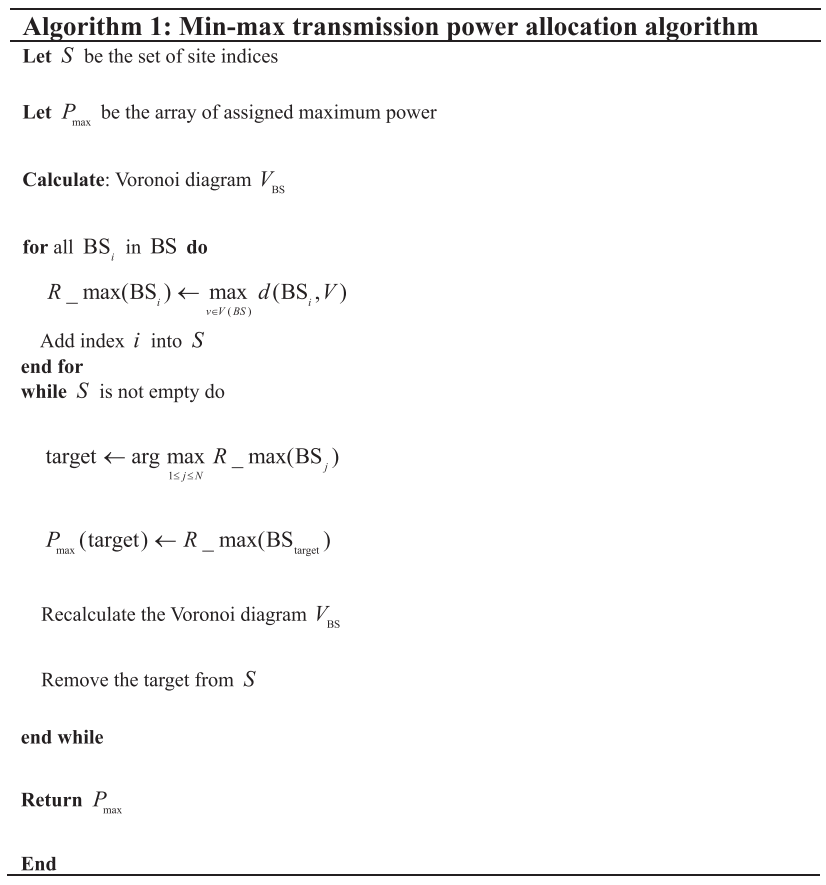

After getting the $P_{\max }\left(B S_{i}\right)$ via the above algorithm, the optimization deployment of the small cell clusters will be determined. Then, we can further consider the cross-tier interferences mitigation issue.

\section{Game theory-based dynamic power control scheme for cross-tier interference mitigation}

With the implementation of Voronoi diagram-based min-max power allocation algorithm, the transmission power limitations for all the small cells are determined. The maximum transmission power of each small cell BS is minimized and the intra-tier interferences are also mitigated, which could improve the system energy efficiency and mobility performances with optimized deployments. But remember that all above analyses are based on the simplified scenario that the cross-tier interferences between the macro cell tier and the small cell tier are temporally ignored. Actually, in the shared spectrum overlaid HetNet deployments, not only the macro cells will cause interferences to the small cells due to their relatively higher transmission power, but also the densely deployed small cells will cause heavy interferences to the macro cells.
Let us take considerations of the cross-tier interferences with the system scenario of several small cell clusters located within the coverage of each macro cell. We assume that there are inter-cell interference coordination (ICIC) schemes implemented in the macro cell tier, such as fractional frequency reuse (FFR); the interferences between different macro cells can be sufficiently avoided [17]. Without loss of generality, we will focus only one macro cell with multiple small cell clusters inside of the macro coverage as Figure 1 showed. Because the small cell clusters will share the spectrum with the co-located macro cell, if we can successfully solve the cross-tier interferences inside one macro cell, we could mitigate the interferences in the whole network due to the ICIC used among different macro cells.

The downlink power control for the above situation will be researched via the game theory approach based on the transmission power limitations already achieved by the min-max power allocation algorithm. The convex pricing-based non-cooperative game will be applied for the macro cell and small cell overlaid HetNet deployment downlink power control. The convex pricing function is an exponential pricing function of each cells' transmission power, which can reflect the crosstier interferences.

Furthermore, in the overlaid HetNet deployment of the macro cell and small cell, the main responsibility for the small cells will be the maximum offloading of higher quality of services (QoS) while the macro cell will mainly handle the high-mobility users or the guaranteed lower QoS. Therefore, without loss of generality, we will assume that the users served by the macro cell will have more priority in the power control-based interference mitigation via the game theory.

Assume that there are $N$ small cell BSs located in the focused macro cell coverage, so, there will be totally $N+$ 1 BSs with the macro cell BS. Each BS serves several users in its coverage. The transmission power of BS $i$ for its served user $j$ is represented by $p_{i, j}$ Watts. The channel gain of the link between the BS $i$ and user $j$ is denoted by $g_{i, j}$ which is calculated from the modified path loss model in $[14,18]$. Specially, we take $i=0$ to represent the macro cell BS and $i=\{1,2, \ldots, N\}$ to denote the small cell BSs. The path loss models for the macro cell and small cell are different [6]. The additive white Gaussian noises at the macro cell BS and the small cell BSs are denoted by $N_{0} \Delta f$. We will use a more simplified form of the $\operatorname{SINR} \gamma_{i, j}$ from Equation 1 of BS $i$ for user $j$ as:

$$
\gamma_{i, j}=\frac{p_{i, j} g_{i, j}}{I_{i, j}}=\frac{p_{i, j} g_{i, j}}{\sum_{n \neq i} p_{n, j} g_{n, j}+N_{0} \Delta f}
$$

where $I_{i, j}$ is the interference power measured by the BS $i$. 


\section{Game model for the dynamic power control scheme}

From the macro cell BS and the small cell BS viewpoint, the power control scheme based on game theory is illustrated as follows. Each BS will choose the transmission power level to maximize its user-serving utility to provide better throughput, which is a typical noncooperative $N+1$-player game. The game model is defined as:

$$
G=\left[N,\left\{p_{i, j}\right\},\left\{u_{i, j}\left(p_{i, j}, \gamma_{i, j} \mid \mathbf{p}_{-\mathbf{i}}\right)\right\}\right]
$$

which represents the downlink power control game via convex pricing. The related definitions are as follows:

a) $i=\{0,1,2, \ldots, N\}$ is the index set of the macro cell and small cells.

b) $p_{i, j}=\left\{p_{i, j} \mid p_{i, j} \in\left[0, p_{\max }\left(B S_{i}\right)\right]\right\}$ is the set of transmission power of the $\mathrm{BS} i$, in which the $p_{\max }$ $\left(B S_{i}\right)$ is the maximum transmission power limitation of BS $i$ obtained by the min-max power allocation algorithm. The maximum power limitation for the macro cell BS $(i=0)$ is set by the ICIC scheme.

c) $u_{i, j}\left(p_{i, j}, \gamma_{i, j} \mid \mathrm{p}_{-\mathrm{i}}\right)$ is the utility function of BS $i$ for the served user $j$, where $\gamma_{i, j}$ is the corresponding received SINR. $\mathrm{p}_{-\mathrm{i}}$ is the vector of transmission power of all BSs other than the BS $i$.

The utility function of the user $j$ with the BS $i$ is defined as the following two equations, which represent the macro cell BS and the small cell BS separately [14].

For the macro cell BS $(i=0)$, the utility function is defined as:

$$
u_{0, j}\left(p_{0, j}, \gamma_{0, j} \mid \mathbf{p}_{-0}\right)=-\left(\gamma_{0, j}-\Gamma_{0, j}\right)^{2}
$$

where $\Gamma_{0, j}$ is the target SINR of the user served by the macro cell.

For the small cell BSs $(i=\{1,2, \ldots, N\})$, the utility function is defined as

$$
u_{i, j}\left(p_{i, j}, \gamma_{i, j} \mid \mathbf{p}_{-\mathbf{i}}\right)=R\left(\gamma_{i, j}, \Gamma_{i, j}\right)-C\left(p_{i, j}, \mathbf{p}_{-\mathbf{i}}\right)
$$

where $R\left(\gamma_{i, j}, \Gamma_{i, j}\right)$ denotes the revenue function and $C\left(p_{i, j}\right.$, $\mathbf{p}_{-\mathbf{i}}$ ) denotes the pricing function for the small cell BSs.

The small cell BS revenue function $R\left(\gamma_{i, j}, \Gamma_{i, j}\right)$ is defined as:

$$
R\left(\gamma_{i, j}, \Gamma_{i, j}\right)=1-e^{-a_{i, j}\left(\gamma_{i, j}-\Gamma_{i, j}\right)}
$$

The revenue function (Equation 12) comes from the bit error rate (BER) expressions in the case of an additive white Gaussian noise (AWGN) channel for different modulation techniques, which is directly related with the throughput performance [19]. The parameter $a_{i, j}$ is a positive factor and its value will be decided by the adopted modulation type. The revenue function (Equation 12) means when the user SINR $\gamma_{i, j} \leq \Gamma_{i, j}$, the revenue of the user will be lower sharply as $\gamma_{i, j}$ decreases and when $\gamma_{i, j}>\Gamma_{i, j}$, the revenue of the user will be increasing slowly as $\gamma_{i, j}$ increases. This revenue function could reflect the user throughput satisfaction level with the QoS requirement target $\Gamma_{i, j}$ for full buffer services.

The pricing function $C\left(p_{i, j}, \mathbf{p}_{-\mathbf{i}}\right)$ is defined as:

$$
C\left(p_{i, j}, \mathbf{p}_{-\mathbf{i}}\right)=\frac{b_{i, j} g_{0, j}}{I_{0, j}}\left(e^{p_{i, j}}-1\right)
$$

which is a convex exponential pricing function of the transmission power $p_{i, j} \frac{b_{i, j} g_{0, j}}{I_{0, j}}$ stands for the interference that the macro cell users suffered from the small cells. The parameter $b_{i, j}$ is a positive factor, which could be adjusted to reflect how severe interferences of the small cells affect the macro cell. Generally, the pricing function is usually defined as the linear function of the transmission power $p_{i, j}$, but in this paper, the interferences from the small cell cluster could be more severe due to the densely overlaid small cell deployments. Therefore, we define the exponential pricing function, which can ensure that the small cells will be penalized when causing serious interference to the guaranteed macro cell users as mentioned above. The convex property of the pricing function is designed to fasten the converging process of finding the Nash equilibrium solution.

Finally, the non-cooperative game model-based power control problem can be formulated as:

$$
\max _{0 \leq p_{i, j} \leq P_{\max \left(B \mathrm{BS}_{i}\right)}} u_{i, j}\left(p_{i, j}, \gamma_{i, j} \mid \mathbf{p}_{-\mathbf{i}}\right), \forall i=0,1,2, \ldots, N
$$

In order to find the transmission power $p_{i, j}$ which can maximize the utility of the above game, we need to find the solution of the corresponding Nash equilibrium. The Nash equilibrium is a steady state which offers a predictable outcome of a game where the game players compete with selfish actions through self-optimization and converge to one point that no player wishes to deviate unilaterally. Moreover, the existence and uniqueness of the possible Nash equilibrium should also be studied. For the proposed game via convex pricing for the power control of the macro cell and small cells, the Nash equilibrium is defined as follows.

Definition 1: Suppose $p_{i, j}^{*}, \forall i=0,1,2, \ldots, N$ is one solution for Equation 14. The $p_{i, j}^{*}$ will be one Nash equilibrium for the proposed non-cooperative game $G$ if for any $\mathbf{p}$, the following conditions are satisfied:

$$
u_{i, j}\left(p_{i, j}^{*}, \gamma_{i, j}^{*} \mid \mathbf{p}_{-\mathbf{i}}^{*}\right) \geq u_{i, j}\left(p_{i, j}, \gamma_{i, j} \mid \mathbf{p}_{-\mathbf{i}}\right), \forall i=0,1,2, \ldots, N
$$


Let us verify the existence and uniqueness of the above Nash equilibrium for the game $G$.

\section{Existence of the Nash equilibrium}

Theorem 1: The Nash equilibrium exists in the game $G=$ $\left[N,\left\{p_{i, j}\right\},\left\{u_{i, j}\left(p_{i, j}, \gamma_{i, j} \mid \mathbf{p}_{-\mathbf{i}}\right)\right\}\right]$ if, for all $\forall i=0,1,2, \ldots, N$ :

a) $p_{i, j}$ is a nonempty, convex, and compact subset of the Euclidean space $\mathbb{R}^{N}$;

b) The utility function $u_{i, j}\left(p_{i, j}, \gamma_{i, j} \mid \mathrm{p}_{-\mathrm{i}}\right)$ is continuous in the $p_{i, j}$ and quasi-concave in the $p_{i, j}$ (Debreu, 1952; Glicksberg, 1952; Fan, 1952).

Proof: Please see Appendix A.

\section{Uniqueness of the Nash equilibrium}

Theorem 2: The Nash equilibrium for the game model via convex pricing has the unique Nash equilibrium point [20].

Proof: Please see Appendix B.

After proving the existence and the uniqueness of the Nash equilibrium for the proposed power control game via convex pricing, the BSs transmission power at the Nash equilibrium point could be regarded as the reasonable solution for the power control problem to maximize the user utility with improving the throughput performance but reducing the cross-tier interferences.

Then, based on the proposed joint Voronoi diagram and game theory-based power control scheme, the optimized deployment approach for the small cell clusters within the heterogeneous network scenario could solve the intra-tier interference problem by minimizing the maximum transmission power of densely deployed small cells. And the outage probability could also be reduced to guarantee the mobility performance. The game theorybased power control scheme with convex pricing functions could mitigate the cross-tier interference while guaranteeing the macro cell users. And the existence and uniqueness of the Nash equilibrium for the proposed game model are verified which provide the feasible solution for the cross-tier power control in the heterogeneous network. We will evaluate the performance via the system-level simulations in the next section.

\section{Simulation evaluations}

In this section, we will analyze the performance of the proposed joint Voronoi diagram and game theory-based power control scheme from the viewpoints of system throughput, cell edge user throughput, outage performance, and energy efficiency. We first present the simulation scenario, followed by the simulation results with observations and performance analyses.

\section{Simulation scenario}

The scenario used in our downlink system-level simulations consists of multi-macro cell BSs and one small cell cluster uniformly distributed within each sector of the macro cell. The small cell cluster is made up of $N$ small cell BSs where $N$ is a variable in the simulation. We employ a 19-hexagonal macro cell model with three sectors per macro cell. Within one sector, there are 60 users in total. Among them, 2/3 of the users are randomly and uniformly distributed within the small cell cluster and $1 / 3$ of the users are randomly and uniformly distributed throughout the macro cell geographical area. Twenty percent are outdoor users and $80 \%$ are indoor users.

To evaluate the most severe case for interference mitigation, a full load and full buffer network is established. Detailed simulation assumptions are largely based on 3GPP LTE-A evaluation methodology [15] for getting more practical simulation results that are comparable with other evaluations. The simulation parameters are summarized in Table 1.

\section{Simulation results and performance analyses}

The results are presented with 1,000 random network instantiations, generated according to the aforementioned simulation parameters. The simulation results of the system throughput, cell edge user throughput, outage probability, and energy efficiency are collected to plot the figures. The following power allocation schemes are used for comparisons. We use the joint power control scheme for the abbreviation of the proposed joint Voronoi diagram and game theory-based power control scheme. The sleep mode-based algorithm for the small cell HetNet proposed in [2,7] and the efficient power control scheme for the HetNet proposed in [23] are compared to show the performances of proposed scheme. For the sleep modebased algorithm, the small cell-controlled sleep mode is applied with the small cell BS that could determine its ON/OFF state according to the user accessing requirements, which has no power control functions. For more fair comparison, the efficient power control scheme for the HetNet with implementation of non-cooperative game model-based power control scheme is simulated as another comparison algorithm. Moreover, the equal power allocation is set as the baseline that each small cell BS is assigned with the fixed RF maximum transmission power of $30 \mathrm{dBm}[15]$.

The simulation results in Figures 3 and 4 show the system throughput and cell edge user throughput versus different deployment densities of small cells. As expected, the best system throughput performance is obtained when small cells perform the proposed joint power control scheme. Not only the system throughput performances are enhanced compared with the other three schemes, but also the cell edge user throughput observed have an 
Table 1 Simulation parameters

\begin{tabular}{lll}
\hline Parameters & Macro cell & Small cell \\
\hline Bandwidth & $10 \mathrm{MHz}$ & $10 \mathrm{MHz}$ \\
RF maximum transmission power & $46 \mathrm{dBm}$ & $30 \mathrm{dBm}$ \\
Path loss model & ITU Uma [referring to Table B.1.2.1-1 in [21]] & ITU Umi [referring to Table B.1.2.1-1 in [21]] \\
Antenna pattern & Three-dimensional (3D) antenna [22] & Omni-directional antenna (horizontal) \\
Antenna gain & $17 \mathrm{dBi}$ & $5 \mathrm{dBi}$ \\
Penetration loss & Outdoor user: $0 \mathrm{~dB}$ & \\
& Indoor user: $20 \mathrm{~dB}+0.5 \mathrm{~d}$ in [21] & \\
Antenna configuration & $2 \mathrm{~T} \times 2$ Rx in DL, 1 T×2 Rx in UL, cross-polarized & \\
UE noise figure & $9 \mathrm{~dB}$ & \\
Inter-site distance macro cell & $500 \mathrm{~m}$ & \\
Minimum inter-site distance small cell & $20 \mathrm{~m}$ & \\
UE speed & $3 \mathrm{~km} / \mathrm{h}$ \\
Small cell numbers in each sector of macro cell & {$[4,10] \times$ cluster number in each sector of macro cell } & \\
Minimum distance (horizontal) & Macro cell BS to user: $35 \mathrm{~m}$ & \\
& Small cell BS to user: $5 \mathrm{~m}$ & \\
\hline
\end{tabular}

obvious improvement. This is because by using Voronoi Diagram based min-max transmission power allocation algorithm, the coverage areas of the small cells are optimized with both the coverage holes and cross-coverage areas, which guarantee the throughput performance especially in the cell edges. The proposed joint power control scheme with game theory solution further reduces the cross-tier interferences and enhances the throughput. Therefore, the cell edge users suffer from less interference, which making their throughput enhanced greatly.

The simulation results in Figure 5 illustrate the system outage probability for the four schemes. The system outage probability is referred to as the fraction of users whose SINR falls below the SINR threshold (-6 dB) [24]. The outage probability can also be adopted to evaluate the user mobility performance because the users located in the cross-coverage area are more frangible for getting guaranteed service, which may cause heavier outage probability in the cell edge. We can observe that the outage probability our scheme provided is lower than that of the efficient power control scheme, sleep modebased algorithm, and equal power allocation algorithm. Since the proposed Voronoi diagram-based transmission power allocation algorithm optimized the coverage area of

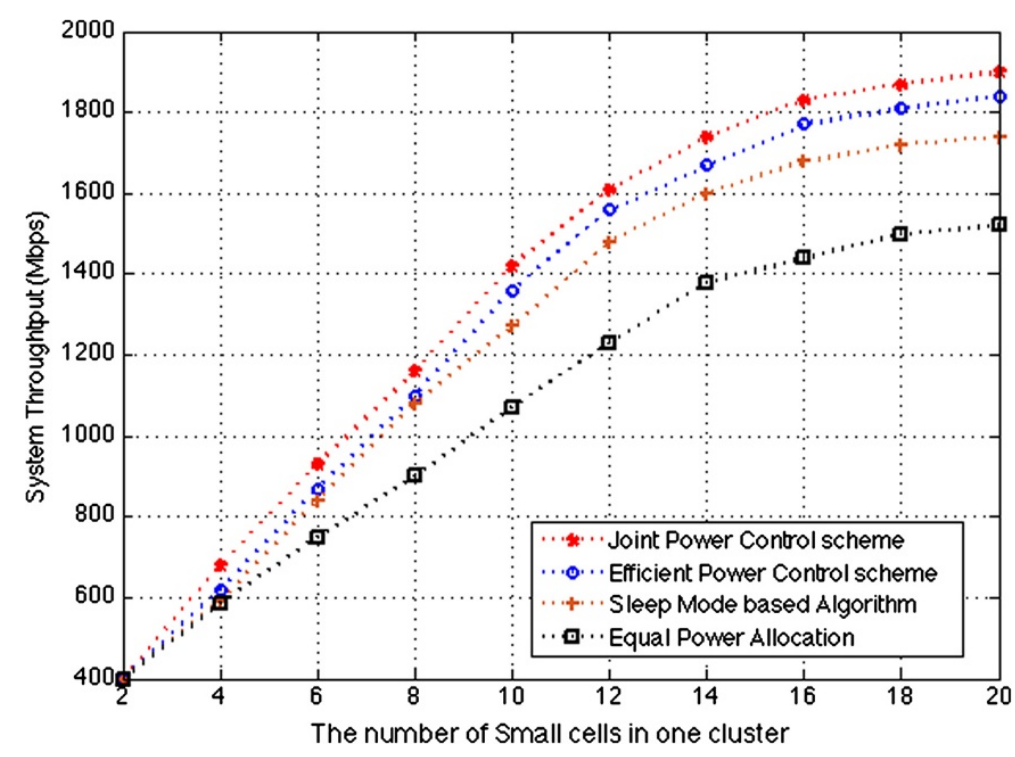

Figure 3 The system throughput. 


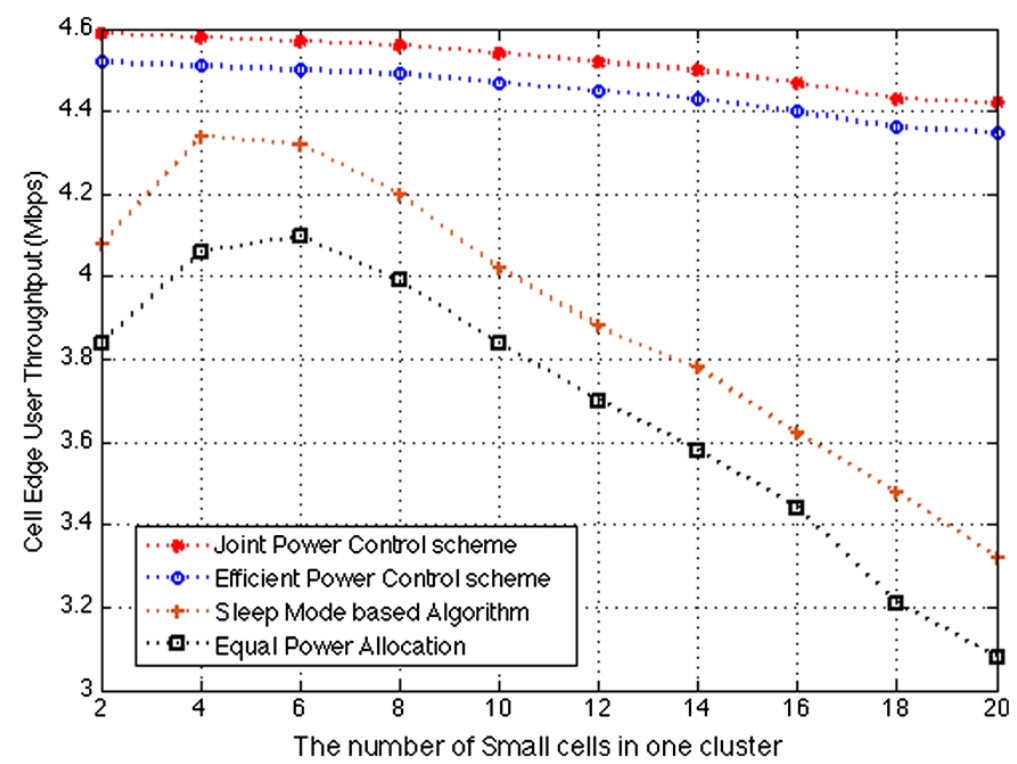

Figure 4 The cell edge user throughput.

the small cells, it led to better coverage ability for the users. Then, the Game Theory based-power control scheme further mitigated the cross-tier interferences, which highlights the cell edge users and enhances their QoS that leads to a decrease of system outage probabilities especially for the dense deployed scenario.

Moreover, we also provide the system energy efficiency as another metric of system performance evaluation. The energy efficiency is expressed by the ratio of system throughput to the whole BS energy consumption as described in Equation 5. According to the simulation results in Figure 6, we can observe that the energy efficiency of our scheme and efficient power control scheme are far better than the sleep mode-based algorithm and equal power allocation algorithm. Besides, as the number of small cells in one cluster increases, the proposed power control scheme provides higher energy savings. The game theory-based power control schemes implemented in these two schemes are the main contribution for the performance enhancements. The proposed joint power

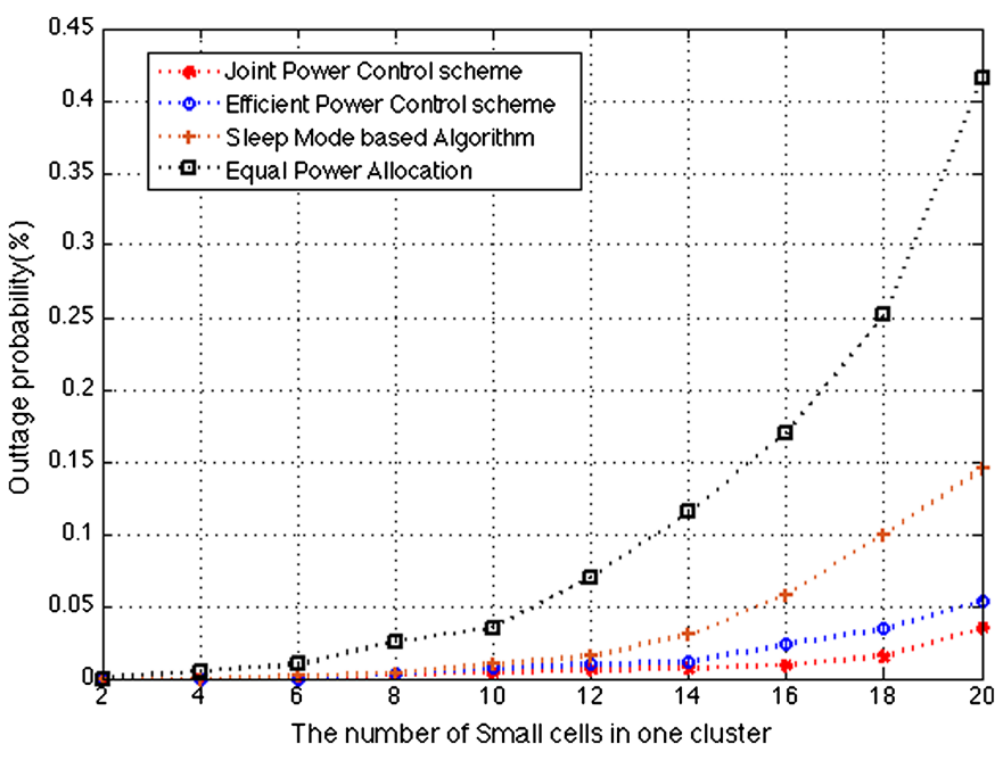

Figure 5 Outage probability performances. 


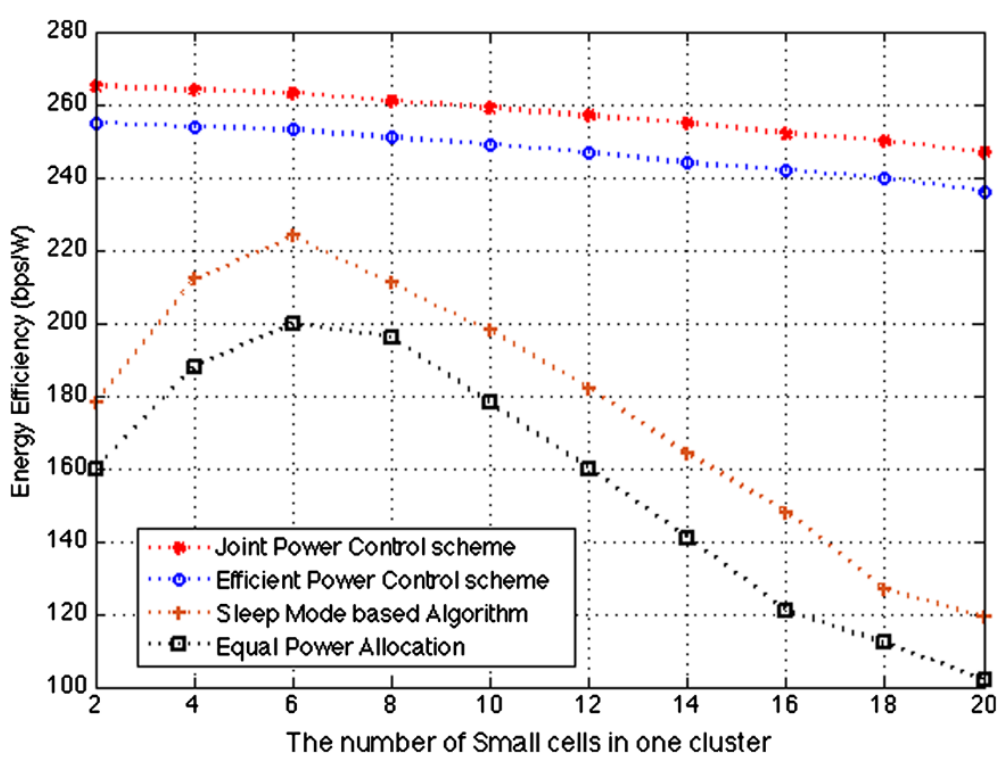

Figure 6 System energy efficiency.

control can further improve the energy efficiency than the efficient power control scheme due to the Voronoi diagram-based min-max small cell transmission power allocation algorithm.

Taking all the above system performances into consideration, we can conclude that the joint Voronoi diagram and game theory-based power control scheme is an effective solution to deploy the small cells in the HetNet networks.

\section{Conclusions}

This article proposes the joint Voronoi diagram and game theory-based power control scheme for the HetNet small cell networks in order to solve the interference and mobility management problems that existed in the overlaid heterogeneous deployments. The joint power control approach is separated into two steps. The first step focuses on the optimization of the small cell cluster deployment planning within the coverage of one macro cell via the Voronoi diagram with implementation of minmax power allocation algorithm. Then, based on the results of the first step, the second step focuses on the cross-tier interferences mitigation through the noncooperative game model with the convex pricing function, by which the existence and uniqueness of the Nash equilibrium are verified, which provide the feasible solution for the cross-tier power control in the heterogeneous network. The system-level simulation results reveal the advantages of the proposed scheme in the system throughput, outage probability, and the energy efficiency. Therefore, we conclude that the proposed scheme is a competitive choice to carry out the small cell deployments in the HetNet networks.

\section{Appendix}

\section{A. Proof of Theorem 1:}

Because $p_{i, j}$ is the transmission power of macro cell and small cell BSs with the strategy space limited by $p_{i, j}=[0$, $\left.p_{\max }\left(B S_{i}\right)\right]$ in the above scheme, the first condition is satisfied.

For any BSs, the first-order partial derivative of the utility function $u_{i, j}\left(p_{i, j}, \gamma_{i, j} \mid \mathbf{p}_{-\mathbf{i}}\right)$ with respect to the $p_{i, j}$ is:

$$
\frac{\partial u_{i, j}}{\partial p_{i, j}}=\frac{a_{i, j} g_{i, j}}{I_{i, j}} e^{-a_{i, j}\left(\gamma_{i, j}-\Gamma_{i, j}\right)}-\frac{b_{i, j} g_{0, j}}{I_{0, j}} e^{p_{i, j}}
$$

The second-order partial derivative is

$$
\frac{\partial^{2} u_{i, j}}{\partial p_{i, j}{ }^{2}}=-\left(\frac{a_{i, j} g_{i, j}}{I_{i, j}}\right)^{2} e^{-a_{i, j}\left(\gamma_{i, j}-\Gamma_{i, j}\right)}-\frac{b_{i, j} g_{0, j}}{I_{0, j}} e^{p_{i, j}}<0
$$

which means that the utility function $u_{i, j}\left(p_{i, j}, \gamma_{i, j} \mid \mathbf{p}_{-} \mathbf{i}\right)$ is a concave function, and the $u_{i, j}\left(p_{i, j}, \gamma_{i, j} \mid \mathbf{p}_{-} \mathbf{i}\right)$ is quasiconcave in the $p_{i, j}$.

Therefore, Theorem 1 has been proved and there exists the Nash equilibrium with the proposed power control game.

\section{B. Proof of Theorem 2:}

Based on the partial derivative of $u_{i, j}\left(p_{i, j}, \gamma_{i, j} \mid \mathbf{p}_{-} \mathbf{i}\right)$ is given as Equation 16, let

$$
\frac{\partial u_{i, j}}{\partial p_{i, j}}=\frac{a_{i, j} g_{i, j}}{I_{i, j}} e^{-a_{i, j}\left(\gamma_{i, j}-\Gamma_{i, j}\right)}-\frac{b_{i, j} g_{0, j}}{I_{0, j}} e^{p_{i, j}}=0
$$


Rearranging Equation 18, we can obtain

$$
\left.p_{i, j}=\min \left\{\begin{array}{c}
{\left[\frac{I_{i, j}}{I_{i, j}+a_{i, j} g_{i, j}}\right.} \\
p_{\max \left(B S_{i}\right)}
\end{array}\left(a_{i, j} \Gamma_{i, j}+\ln \frac{a_{i, j} g_{i, j}}{b_{i, j} g_{0, j}}\right)\right]^{+}\right\}
$$

Then with the iterations,

$$
p_{i, j}^{(k+1)}=\min \left\{\left[\begin{array}{l}
\frac{p_{i, j}^{(k)}}{p_{i, j}^{(k)}+a_{i, j} \gamma_{i, j}^{(k)}} \\
p_{\max \left(\mathrm{BS}_{i}\right)}
\end{array}\left(a_{i, j} \Gamma_{i, j}+\ln \frac{a_{i, j} g_{i, j}}{b_{i, j} g_{0, j}}\right)\right]^{+}\right\}
$$

To provide a general framework for showing the convergence of power control algorithms, the standard interference function $p=f(p)$ will be introduced. One function is proved to be standard if it satisfies the following properties:

a) Positivity: $f(p)>0$;

b) Monotonicity: if $p \geq p^{\prime}$, then $f(p) \geq f\left(p^{\prime}\right)$;

c) Scalability: for all $\alpha>1, \alpha f(p)>f(\alpha p)$.

For the macro cell BS, the above three properties are obviously satisfied. For the small cell BSs, we will prove the above properties as follows.

Assume

$$
f_{i, j}(p)=\min \left\{\left[\begin{array}{l}
\frac{p_{i, j}^{(k)}}{p_{i, j}^{(k)}+a_{i, j} \gamma_{i, j}^{(k)}} \\
p_{\max (\mathrm{BS})}
\end{array}\left(a_{i, j} \Gamma_{i, j}+\ln \frac{a_{i, j} g_{i, j}}{b_{i, j} g_{0, j}}\right)\right]^{+}\right\}
$$

which is obvious that $p_{i, j} \geq 0$, the positivity property is satisfied.

Rearranging the above equation, we will get

$f_{i, j}(p)=\min \left\{\left[\frac{1}{1+a_{i, j} \gamma_{i, j}^{(k)} / p_{i, j}^{(k)}}\left(a_{i, j} \Gamma_{i, j}+\ln \frac{a_{i, j} g_{i, j}}{b_{i, j} g_{0, j}}\right)\right]^{+}\right\}$

If $p \geq p^{\prime}$, then $\frac{\gamma}{p} \leq \frac{\gamma}{p^{\prime}}$ and $\frac{1}{1+\frac{\gamma}{p}} \geq \frac{1}{1+\frac{\gamma}{p^{\prime}}}$, then $f_{i, j}(p) \geq f_{i, j}\left(p^{\prime}\right)$.

The monotonicity property is verified.

To prove the last property, we define a function $F(\alpha)=$ $\alpha f_{i, j}(p)-f_{i, j}(\alpha p)$, whose first-order partial derivative with respect to $\alpha$ is:

$$
\frac{\partial F(\alpha)}{\partial \alpha}=\frac{\alpha(\alpha-1)[(\alpha+1) p+2 \gamma] p \gamma}{(p+\gamma)^{2}(\alpha p+\gamma)^{2}}\left(a_{i, j} \Gamma_{i, j}+\ln \frac{a_{i, j} g_{i, j}}{b_{i, j} g_{0, j}}\right)
$$

Then, for all $\alpha>1, \frac{\partial F(\alpha)}{\partial \alpha}>0$, and $F(1)=0$, we can get $F(\alpha)>F(1)$.

This means for all $\alpha>1, \alpha f_{i, j}(p)>f_{i, j}(\alpha p)$. Therefore, the scalability property is satisfied. The proofs of Theorem 2 are completed.

\section{Competing interests}

The authors declare that they have no competing interests.

\section{Acknowledgments}

This paper is supported by the Nature and Science Foundation of China under Grants No. 61471068, 61325006; Beijing Nova Programme No. Z131101000413030; National High Technology Research and Development Program of China No. 2014AA01A701; and National Major Project No. 2013ZX03003002-002. The authors also thank the reviewers and editors for their review and comments.

\section{Author details}

${ }^{1}$ National Engineering Laboratory for Mobile Network Security, Beijing University of Posts and Telecommunications, Beijing 100872, China. ${ }^{2}$ Network Technology Research Institute, China United Network Communications Corporation Limited, Beijing 100044, China.

Received: 30 June 2014 Accepted: 18 November 2014 Published: 6 December 2014

\section{References}

1. L Hanzo, H Haas, S Imre, D O'Brien, M Rupp, L Gyongyosi, Wireless myths, realities, and futures: from $3 \mathrm{G} / 4 \mathrm{G}$ to optical and quantum wireless. Proc. IEEE 100, 1853-1888 (2012)

2. A Imran, B Federico, $H$ Lester, SLEEP mode techniques for small cell deployments, Communications Magazine. IEEE 49(8), 72-79 (2011)

3. JG Andrews, Seven ways that HetNets are a cellular paradigm shift. Commun. Mag. IEEE 51(3), 136-144 (2013)

4. Huawei, HiSilicon, CATR, RP-121764, LTE Small Cell Enhancement - Physical Layer SID (3GPP, TSG-RAN Meeting \#58, Barcelona, 2012)

5. J Hoydis, M Kobayashi, M Debbah, Green small-cell networks. Veh. Technol. Mag. 6(1), 37-43 (2011)

6. 3GPP, TR36.872, Small cell enhancements for E-UTRA and E-UTRAN - Physical layer aspects (Release 12) (3GPP, Sophia-Antipolis, 2013)

7. H Claussen, IHLTW Ashraf, Dynamic idle mode procedures for femtocells. Bell. Labs. Tech. J. 15(2), 95-116 (2010)

8. Ericsson, ST-Ericsson, R2-130416, Small cell challenges and benefits of dual connectivity (3GPP TSG-RAN WG2 \#81, Malta, 2013)

9. Ericsson, ST-Ericsson, R2-131678, Further discussions on UL/DL split (3GPP TSG-RAN WG2 \#82, Fukuoka, 2013)

10. V Chandrasekhar, JG Andrews, T Muharemovict, Z Shen, A gatherer, power control in two-tier femtocell networks. IEEE Trans. Wireless Commun. 8, 4316-4328 (2009)

11. M Ahmed, P Mugen, I Ahmad, L Yuan, M Abana, Stackelberg game based optimized power allocation scheme for two-tier femtocell network. IEEE Wirel. Commun. Netw. Conf. 1-6 (2013)

12. M Mhiri, VS Varma, M Le Treust, S Lasaulce, A Samet, On the benefits of repeated game models for green cross-layer power control in small cells (2013 First International Black Sea Conference on Communications and Networking, IEEE, New York, 2013), pp. 137-141

13. M Hkimi, R Langar, S Secci, R Boutaba, G Pujolle, Demands rescaling for resource and power allocation in cooperative femtocell networks (2013 IEEE International Conference on Communications (ICC), IEEE, New York, 2013), pp. 6235-6239

14. W Li, W Zheng, S Tao, X We, Distributed power control and pricing for two-tier OFMDA femtocell networks using fictitious game. IEEE Wirel. Commun. Netw. Conf. 470-475 (2013) 
15. Huawei, HiSilicon, CATR, CMCC, R1-130744, WF on evaluation assumptions for SCE physical layer (3GPP TSG RAN WG1 \#72, Malta, 2013)

16. M De Berg, M Overmars, O Cheong, M Van Kreveld, Computational Geometry: Algorithms and Applications (Springer, New York, 2010), pp. $147-170$

17. X Zhikun, GY Li, C Yang, X Zhu, Throughput and optimal threshold for FFR schemes in OFDMA cellular networks. Trans. Wireless Commun. 11(8), 277-2785 (2012)

18. J Liu, W Zheng, W Li, X Wang, Y Xie, X Wen, Distributed uplink power control for two-tier femtocell networks via convex pricing. IEEE Wirel. Commun. Netw. Conf. 458-463 (2013)

19. CU Saraydar, NB Mandayam, DJ Goodman, Efficient power control via pricing in wireless data networks. IEEE Trans. Commun. 50(2), 291-303 (2002)

20. RD Yates, A framework for uplink power control in cellular radio systems. IEEE J. Sel. Areas Commun. 13(7), 1341-1347 (1995)

21. 3GPP, TS 36.814 V9.0.0, Further Advancements for E-UTRA Physical Layer Aspects (3GPP, Sophia-Antipolis, 2010)

22. 3GPP, TR 36.873 v2.0.0, Study on 3D channel model for LT (3GPP, Sophia-Antipolis, 2014)

23. $Y M a, T L V, L$ Yueming, Efficient power control in heterogeneous femtomacro cell networks. IEEE Wirel. Commun. Netw. Conf. 2515-2519 (2013)

24. 3GPP, TR 36.942 v10.2.0, E-UTRA Radio Frequency (RF) System Scenarios (3GPP, Sophia-Antipolis, 2012)

doi:10.1186/1687-1499-2014-213

Cite this article as: Xu et al:: Joint Voronoi diagram and game

theory-based power control scheme for the HetNet small cell networks.

EURASIP Journal on Wireless Communications and Networking

2014 2014:213.

\section{Submit your manuscript to a SpringerOpen ${ }^{\circ}$} journal and benefit from:

- Convenient online submission

- Rigorous peer review

- Immediate publication on acceptance

- Open access: articles freely available online

- High visibility within the field

- Retaining the copyright to your article 